\title{
Stefan Immerfall \\ Über die Euro-Krise zur Fiskalunion? Mögliche Lehren für europäische Integration
}

Durch den Schuldenschnitt Griechenlands vom 9. März 2012 sollte ein Staatsbankrott, wie er - nicht nur - in der Geschichte Griechenlands schon so oft vorkam, ${ }^{1}$ vermieden werden. Es war der größte Schuldenschnitt der Geschichte. Zudem führte erstmals in der Geschichte der Währungsunion eines der Euro-Mitglieder rückwirkend Umschuldungsklauseln in seine Anleiheverträge ein (mittels des sogenannten Collective Action Clauses). Spätestens jetzt musste jedem Gläubiger klar sein, dass er sein in europäische Staatsanleihen investiertes Geld tatsächlich verlieren kann.

Die Euro-Krise und vor allem die vielfältigen Versuche der Euro-Retter, sie in den Griff zu bekommen, sollten weitere Überraschungen bereit halten. Deren derzeit letzte (März 2013) ist die >Rettung Zypernsく, welche deutlich machte, dass auch Bankkunden zur Kasse gebeten werden können, wenn Geldinstitute saniert oder abgewickelt werden. Im Zweifel sind die Sparguthaben selbst von Kleinsparern keineswegs so sicher, wie die Bundeskanzlerin behauptet. Wie jeder Versicherungskunde weiß, sind Garantien besonders dann sicher, wenn man sie nicht braucht.

Aber schon die griechischen Hilfspakete ließen die Leitmotive erkennen, die auch spätere Rettungsmaßnahmen kennzeichneten: die Zahlungsunfähigkeit zahlungsunfähiger Staaten vermeiden, ssystemrelevante< Banken retten, vor allem aber die Währungsunion bewahren. Vom Rettungspaket für Griechenland sahen Herr und Frau Papadopoulos nicht viel; ein großer Teil der EU- und IWF-Gelder flossen auf ein Sperrkonto, aus dem bestehende Kredite bedient werden. Ferner konnten sich die großen Banken und Versicherungen vor dem Schuldenschnitt eines Teils ihrer faulen Kredite zu Lasten der Europäische Zentralbank entledigen und im Zuge des Schuldenschnitts wird der größere Teil der verbleibenden Altschulden in die öffentliche Hand des Rettungsschirms übertragen. Von daher relativiert sich die Behauptung, die Kreditwirtschaft hätte substantielle Beträge zum Schuldenschnitt geleistet. Schließlich wäre sie bei einem Komplettausfall leer ausgegangen.

Auch ein Jahr und mehrere Hilfstranchen später besteht an der Entschlossenheit der maßgeblichen europäischen Eliten, den Euro zu retten, weiterhin kein Zweifel. »But there is another message I want to tell you. Within our mandate, the ECB is ready to do

1 Carmen M. Reinhart, / Kenneth S. Rogoff, This Time Is Different: Eight Centuries of Financial Folly, Princeton, NJ 2009. 
whatever it takes to preserve the euro. And believe me, it will be enough «. ${ }^{2}$ Mit diesen Worten signalisierte der Präsident der Europäischen Zentralbank den Märkten, dass es unklug wäre, auf ein rasches Auseinanderbrechen der Euro-Zone zu spekulieren. Die Renditen der südeuropäischen Staatsanleihen sanken daraufhin rasch, und das Vertrauen der Märkte in den >Draghi-Put< hält so weit an, dass selbst Italien-Wahlen und ZypernKrise die Risikoprämien für die südeuropäischen Schuldendienst nur wenig erhöhten. ${ }^{3}$

Inzwischen sind durchaus Lichtblicke zu sehen. Die Lohnstückkosten der Krisenländer gingen im Vergleich zum europäischen Durchschnitt zurück, die Defizite im Außenhandel sind gesunken, der Anstieg der Staatsdefizite vorerst gebremst, Portugal und Irland sind erfolgreich an den an internationalen Kapitalmarkt zurückgekehrt. Zu Recht wird darauf hingewiesen, dass bei den Wahlen in den sechs Krisenländern (Irland, Portugal, Griechenland, Spanien, Zypern und Italien) zwar die jeweils amtierende Regierung abgewählt wurde. Noch stärker muss aber betont werden, dass in keinem dieser Länder Parteien an die Macht kamen, die Reformen grundsätzlich ablehnten. Die Lebensumstände für die Bevölkerungen, die für die von ihren `begüterten Klassen ${ }^{4}$ eingegangenen Schuldverpflichtungen herangezogen werden, bleiben aber dramatisch.

Es wurde also Zeit gewonnen. Es fragt sich nur: Zeit für was? Das Ziel der maßgeblichen politischen Eliten Europas dürfte etwa wie folgt zu beschreiben sein: Zurückführen der Staatschulden auf ein tragfähiges Maß und Gewinnung der Wettbewerbsfähigkeit der Peripherieländer soweit, dass innereuropäische Transferzahlungen erträglich, sprich politisch vermittelbar bleiben. Selbst wenn man dieses Ziel für erreichbar halten sollte, stehen erhebliche Übergangshürden im Weg. Darauf nimmt Abschnitt 2 Bezug. Ein alternativer Entwurf fordert einen raschen Souveränitätsverzicht der Nationalstaaten, ob mit oder gegen ihre Bürger, um die Übergangsproblematik abzukürzen (Abschnitt 3). Demgegenüber werden die gesellschaftlichen Grenzen des europäischen Integrationsprojekts herausgearbeitet (Abschnitt 4). Zunächst gehe ich kurz auf den Hintergrund der Eurokrise ein (Abschnitt 1).

2 Speech by Mario Draghi, President of the European Central Bank at the Global Investment Conference in London. 26 July 2012, [http://www.ecb.int/press/key/date/2012/html/sp120726 .en.html; 28.3.2013].

3 Es bedarf keiner Verschwörungstheorie, um das zeitweilige Herdenverhalten von Finanzakteuren zu erklären. Spekulanten wollen Geld verdienen, ob mit oder gegen den Euro. Diese Wetten können auch daneben gehen. Selbst ein John Paulson, der als einer der Ersten gegen den amerikanischen Hypothekenmarkt spekulierte und damit Milliarden verdiente, lag mit seiner Strategie schief, über Leerverkäufe von Eurostaaten-Anleihen und Käufen von Kreditausfallsversicherungen europäischer Krisenländer auf das Auseinanderbrechen der Währungsunion zu setzen, während andere Hedge-Fonds, die auf einen Schuldenrückkauf Griechenland spekulierten, hervorragend verdienten. Vgl. Handelsblatt, 10.1.2013, "Herbe Verluste für Superinvestor Paulson" [http://www.handelsblatt.com/finanzen/fonds/nachrichten/hedge-fonds-herbe-verluste-fuersuperinvestor-paulson/7605246.h tml; 28.3.2013] und Frankfurter Allgemeine Zeitung, 19.12.2012, „Hedgefonds verdient halbe Milliarde Dollar mit Anleihenrückkauf“ [http://www .faz.net/aktuell/finanzen/anleihen-zinsen/griechenland-hedgefonds-verdient-halbe-milliardedollar-mit-anleihenrueckkauf-11999071.html; 28.3.2013].

4 Wolfgang Streeck, Gekaufte Zeit. Die vertagte Krise des demokratischen Kapitalismus, Berlin 2013, S. 221. 


\section{Der Euro: von einer Erfolgsgeschichte zum Sprengsatz für die Union}

Im Januar 1999 wurde der Euro als Buchgeld aus der Taufe gehoben, im Jahr zuvor die Europäische Zentralbank (EZB) als dessen Hüterin. Am 1. Januar 2002 war der Euro als Bargeld zu erhalten. Am 1. Januar 2001 wurde Griechenland zwölftes Mitglied der Währungsunion, die inzwischen aus 17 europäischen Ländern besteht. Dass der Realitätsgehalt der von Griechenland damals gemeldeten Zahlen zu wünschen übrig ließ, war der Kommission und den Finanzministern bewusst, wenn auch wohl nicht das Ausmaß der Irreführung. Griechenland aber war »klein« und schien auf dem richtigen Weg.

Die Einführung des Euros kann zunächst als Gegenleistung für die Zustimmung zur deutschen Einheit verstanden werden: Die Deutschen geben die D-Mark auf und zeigen damit, dass sie auch wiedervereinigt gute Europäer bleiben wollen. Wichtiger war aber, dass die Gemeinschaftswährung die folgerichtige » Krönung « des Binnenmarkts darstellt. Sie sollte das innereuropäische Wirtschaftsleben stimulieren und - ganz nebenbei - eine globale, zum US-Dollar alternative Leitwährung schaffen. Klar war, dass die Voraussetzungen für eine Währungsunion nicht optimal waren. Allerdings wurde ein Schub zugunsten der wirtschaftlichen und sozialen Konvergenz erwartet. Und gegen die Gefahr, dass sich ein Land auf Kosten eines anderen verschulden würde, hatten die Hartwährungsländer - allen voran Deutschland - mit den Maastricht-Kriterien und dem Haftungsausschluss (Art. 125 AEUV) vorgebeugt.

Das Kalkül, Konvergenz über regional unterschiedliche Wachstumswirkungen herzustellen, ist nach Einführung der gemeinsamen Währung auch lange aufgegangen. Der Euro ist international akzeptiert, sein Außenwert stabil, der Binnenmarkt gewachsen, die Inflation niedrig (auch in den ehemaligen Hochinflationsländern). Vor allem aber hatten sich die Zinsen einander stark angenähert. Der Zinsaufschlag, den die südeuropäischen Länder gegenüber den früheren Hartwährungsländern zahlen mussten, ging um 80 bis 90 Prozent zurück. Hinzu kamen die niedrigen EZB-Referenzzinsen. ${ }^{5}$ Beides zusammen wirkte in den Peripherieländern wie ein Konjunkturprogramm. Dies war auch so gewollt, denn diese Länder sollten ja aufholen.

Ein beträchtlicher Teil der quasi subventionierten Kredite floss in den Konsum bzw. in Immobilien (namentlich in Spanien und Irland). Die Reallöhne und Lohnstückkosten stiegen dabei deutlich stärker an als in den produktiveren Ländern. Weil diese Länder in den Genuss deutlich niedriger Zinsen kamen, konnten höhere Staatsschulden zunächst leichter getragen werden. Offenbar vertrauten die Investoren auf einen Haftungsverbund, insbesondere nach dem umstrittenen Beschluss der Finanzminister von 2003, mit dem sie das Defizitverfahren gegen die `Sünder $<$ Deutschland und Frankreich aussetzten. Doch nach der Finanzkrise von 2008 stiegen die Staatsverschuldungen wegen der Rettung der nationalen Bankensysteme und den umfangreichen Konjunkturprogrammen dra-

5 Diese waren aber für Deutschland nach der Vereinigungskrise zu hoch und trugen zur hohen Arbeitslosigkeit und zur starken Lohnzurückhaltung bei. 
matisch. ${ }^{6}$ Was wir heute sehen, sind die Aufräumarbeiten im Gefolge eines neoliberalen, deregulierten Finanzmodells. In dessen Logik haben die Finanzmarktakteure ihre Kredithebel zunehmend ausgeweitet. Der Finanzsektor hat einen immer größeren Anteil an der wirtschaftlichen Wertschöpfung erlangt. Den von seinen Akteuren eingegangenen Risiken standen exorbitante Gewinne und Boni gegenüber. Da diese Risiken wechselseitig abgesichert und dank der vielen Produktinnovationen der Finanzmarktindustrie breit gestreut waren, würden sie indes kein Problem darstellen, zeigte sich der damalige Präsident der amerikanischen Zentralbank, Alan Greenspan, überzeugt, der damit die wohl vorherrschende ökonomische Lehre wiedergab.

Wenn in dieser Situation die Markteilnehmer - aus welchem Grund auch immer - ihre Risiken zurückfahren wollen, steigen die von den Kreditgebern verlangten Sicherheiten und Risikoaufschläge rasant an. Das kann zu einer sich selbst verstärkenden Abwärtsspirale führen, die nur durch externe, staatliche Eingriffe unterbrochen werden kann. Tatsächlich begannen Anleger Staatsanleihen angesichts der seit Beginn der Finanzkrise noch einmal stark gestiegenen öffentlichen Verschuldung von immer mehr Ländern neu zu bewerten und - als Folge der Neubewertung - nur noch gegen höhere Zinsen abzunehmen. Steigende Risikoaufschläge machten die Refinanzierungskosten der despektierlich >PIIGS $<$ genannten Länder ${ }^{7}$ allmählich untragbar, was den Vertrauensverlust zusätzlich beschleunigte. Die Gefahr der Staatsinsolvenz nahm vor allem für Griechenland rasch zu. Eine solche sollte aber vermieden werden, da die europäischen Regierungschefs ihre Kosten und Nebenwirkungen fürchteten. Das führte schließlich zu den verschiedenen Rettungspaketen (der ersten Griechenlandhilfe im April 2010, dem Rettungsschirm im Mai 2010, den Europäischen Stabilisierungsmechanismen, der Europäischen Finanzstabilisierungsfazilität mit aufgestockten Garantiesummen und Kredithebeln im Juli bzw. Oktober 2011, dem Europäischen Stabilisierungsmechanismus ESM vom Januar 2012 und der Notenbankgarantie vom Juli 2012). Ein Ende ist nicht abzusehen.

Auch wenn der Auslöser der Euro-Finanzkrise die Krise des Finanzkapitalismus war, für dessen Stabilisierung die Staaten gewaltige Summen aufbieten mussten, sind doch auch institutionelle Faktoren der Europäischen Union und der Eurozone zu beachten. Denn obwohl das Staatsdefizit der USA in Prozent der Wirtschaftsleistung mehr als doppelt so hoch ist wie das der Eurozone und obwohl einzelne Bundesstaaten ihren Zahlungsverpflichtungen nicht nachgekommen sind, zweifelt niemand an der Zukunft des Dollars. ${ }^{8}$ Trotz des periodisch auftretenden politischen Stillstands gibt es handlungsfähige Zentralinstanzen und trotz der regionalen Vielfalt ist die nationale Einheit der Vereinigten Staaten nie in Gefahr.

6 Vor der Finanzkrise waren vielmehr die Staatsdefizite und Staatsdefizit in einigen der heutigen Krisenländer ausgesprochen gering; vgl. T. D. Wilet / C. Wihlborg, "Varieties of European Crisis«. in: Gerard Caprio (Hg.), Handbook of Safeguarding Global Financial Stability, Boston, S. 309-322.

7 Portugal, Irland bzw. Italien, Griechenland. Spanien.

8 So auch, entgegen des reißerischen Untertitels, Barry Eichengreen, Exorbitant Privilege: The Rise and Fall of the Dollar, Oxford 2011. 
Ganz anders die Europäische Union. Hier ist die Legitimations- und die Organisationsbasis weiterhin national. ${ }^{9}$ Zudem gehen Unterschiede in den kulturellen Merkmalen und in der sozioökonomischen Entwicklung oft mit nationalen Grenzen einher. ${ }^{10}$ Deshalb werden europäische Konflikte bisher weiterhin regelmäßig national ausgedeutet. Stets besteht die Gefahr, dass (national-)räumliche Unterschiede zu Lasten der Einheit politisiert werden. Im Rahmen eines Nationalstaates gibt es mehr - siehe Deutschland mit seinen Auseinandersetzungen um den Finanzausgleich - oder minder (Spanien, Belgien) gute Chancen, solche Konflikte einzuhegen. Im europäischen Kontext hingegen werden Staatsvölker ein Stück weit zu >Marktvölkern ${ }^{11}$ - nämlich im Rahmen der Währungsunion aneinander gekettete Schuldner- und Gläubigerbevölkerungen.

\section{Die Konfliktdynamik der Rettungsroutine}

Im Europäischen Gemeinschaftssystem zwingt der Konsolidierungskurs die Mitgliedsländer in spieltheoretisch unvermeidbare Konfliktsituationen. Vor allem deshalb, weil im Euroraum die Position als Geberland im EU-Haushalt und als Gläubigerland in der Euro-Rettung einerseits, als Empfänger-, bzw. Schuldnerland andererseits mehrheitlich übereinstimmt und somit dem Konvergenz und Kohärenzziel dient. Es handelt sich somit um >overlapping cleavages<, die einer besonderen Gemeinschaftsbildung abträglich sind. ${ }^{12}$ Insofern würde ein Beitritt der aufstrebenden Länder Mittelosteuropas, vor allem Polens, zur Einheitswährung die Situation entschärfen, da sie Nettoempfänger, aber keine Schuldnerländer sind.

Wir haben es eben nicht mit einem seinfachen $\mathrm{Zwei}$-Personen-Spiel von Gläubiger und Schuldner zu tun, in der die Beteiligten die optimale Höhe des Schuldenverzichts aushandeln. (Optimal in dem Sinne, dass der Schuldner durch einen Teilerlass in die Lage versetzt werden soll, möglichst viel seiner Schulden zu bedienen.) Vielmehr gilt es in der Währungsunion zugleich die Regeln des Haftungsverbunds zu bestimmen. Es versteht sich, dass die Schuldnerländer einen möglichst großen Anteil der Hilfen und Gewährleistungen in einen gemeinsam zu bewirtschaftenden Pool überführen möchten. Gleichzeitig werden sie dafür eintreten, dass die Regeln der Poolbewirtschaftung möglichst >demokratisch`, also unabhängig von der Herkunft der zur Verfügung gestellten Res-

9 Stefan Immerfall, »Political unification and the purported European Society. On the social basis of European integration « in: Franz Höllinger und Markus Hadler (Hg.), Crossing Borders, Shifting Boundaries. National and Transnational Identities, Frankfurt / New York 2012, S. 221-236.

10 Werner Abelshauser / David Gilgen (Hg.), Kulturen der Weltwirtschaft, Göttingen 2012.

11 Dies wird in der ansonsten höchst luziden Analyse von Wolfgang Streeck, Gekaufte Zeit, aaO. (FN 4), S. 117-139, übersehen: zwar ist das >Marktvolk< international, ungebunden und mobil, jedoch sind Rahmen der europäischen Schuldenpolitik Transfers auch als Transfers zwischen - sicherlich auch klischeehaft und ideologisch konzipierten - Nationen zu sehen und nicht nur als Tribute gegen Dritte (die internationalen Finanzmärkte). Das macht die Hilfspakete so gefährlich für den europäischen Integrationsgedanken.

12 Stein Rokkan, Staat, Nation und Demokratie in Europa, hg. u. eingel. v. Peter Flora, Frankfurt/M. 2000. 
sourcen gestaltet werden sollten. Da mit der Poolausweitung auch ein Machtzuwachs der EU-Institutionen verbunden ist, haben die Netto-Zahler in dieser Forderung mächtige Verbündete in Brüssel. Deshalb haben die Geberländer neben dem Allmende-Problem zusätzlich ein Koalitions-Problem zu bewältigen. So tritt beispielsweise eine SuperKoalition von FDP, CDU, SPD und Grünen-Mitgliedern des Europäischen Parlaments - von nationaler Herkunft und politische Gesinnung weitgehend unbeeinflusst - für die Ausweitung des EU-Haushalts ein: »Entschlossen werden wir für einen Haushalt kämpfen, der dazu beiträgt, Europa aus der Krise, zu nachhaltigem Wachstum und neuer Wettbewerbsfähigkeit zu führen. Sollte das Ratsangebot keine gute Lösung für Europa darstellen, wird es im Europäischen Parlament auch nicht die nötige absolute Mehrheit geben. ${ }^{13}$

Umgekehrt werden die Nettozahler auf möglichst mehrheitsunabhängige Regelvereinbarungen drängen. Doch auch mehrheitsunabhängige Regelvereinbarungen müssen einstimmig durchgesetzt werden. Schon allein, weil die grundsätzliche Asymmetrie zwischen Mitbestimmungsrechten und Finanzierungsbeiträgen bei allen europäischen Überwachungsmechanismen bestehen muss, sollte man von solchen vertraglichen Regeln nicht zuviel erwarten. Ferner werden die Nettozahler rasche und möglichst unumkehrbare Maßnahmen fordern. Dafür sprechen die Lehren aus der Geschichte politisch-ökonomischer Reformen. ${ }^{14}$ Sie sind oft erst in Krisensituationen durchsetzbar und nicht in guten Zeiten, in denen sie am wenigsten schmerzhaft wären. Außerdem ist es einfacher, harsche Auflagen zu lockern als weiche Auflagen zu verschärfen.

Schon diese kleine Überlegung zeigt dreierlei: Erstens ist die Position der Schuldnerländer im Rahmen der Euro-Rettung stärker als ein erster Blick nahelegen würde. Zweitens - und wichtiger - zwingen die Maßnahmen zur Bewahrung der Fiskalunion ihre Mitglieder beständig und ungewollt in nichtkooperative Situationen. Dies erfolgt im Unterschied $\mathrm{zu}$ anderen Integrationsprozessen, namentlich dem Binnenmarktprogramm, welches sich eher als kooperative Interaktion deuten lässt, wenn beispielsweise durch wechselseitigen Subventionsverzicht alle Mitgliedsländer profitieren. Das gilt für das gemeinschaftlich vereinbarte Ziel - tragbarer Schuldenstand ohne weitere Schuldenschnitte und wettbewerbsfähige Südländer - nicht. Drittens handelt es sich bei der Währungsunion-Rettung um ein „Spiel gegen die Zeit“. Ihre Früchte werden in einer mehr oder minder fernen Zukunft geerntet, während in der Gegenwart den Bürgern immer härtere Auflagen zugemutet werden. Deshalb könnte die schon von Adam Przeworski

13 Frankfurter Allgemeine Zeitung vom 29.1.2013. Die MdEPs verweisen darauf, dass der EU Finanzrahmen "nur 1\% der EU-Wirtschaftskraft" umfasse und Deutschland der Hauptprofiteur des Binnenmarkts sei. Mit dieser Argumentation könnte man sagen: je mehr Nettozahlung desto besser. Abgesehen davon, dass dieses eine Prozent absolut gut 20 Milliarden Euro jährlich ausmacht, müsste doch auch nach den Erfolgskriterien der europäische Agrar- und Strukturfonds gefragt werden. Die regionalen, wirtschaftlichen und sozialen Unterschiede wurden jedenfalls nicht verringert und an die grandiosen Versprechungen der Lissabon-Strategie will man sich erst gar nicht mehr erinnern (Stefan Immerfall, Europa - politisches Einigungswerk und gesellschaftliche Entwicklung. Wiesbaden 2006, S. 111).

14 Helmut Wiesenthal, Gesellschaftsstenerung und gesellschaftliche Selbststenerung. Eine Einfübrung, Opladen 2006. 
(1980) für den wenig wahrscheinlichen, obgleich eigentlich möglichen Sozialismus analysierte Übergangsfalle zuschnappen. Selbst wenn man die EU-Zielvorstellung für realistischer halten sollte als die Hoffnung auf einen demokratischen Sozialismus, stellt sich für die Bürger der Peripherieländer auch hier die Frage, wie lange sie mit und für den Euro durch das »Tal der Tränen « für eine goldene, aber ungewisse Zukunft gehen wollen.

Desweiteren kommt hinzu: Für beide Ländergruppen ist es gute Verhandlungsstrategie, wenn sie auf eine europakritische Opposition zu Hause verweisen können, um damit mehr Kompromissbereitschaft von der jeweils anderen Seite einzufordern. So sollte der Zugewinn der griechischen Linkspartei Syriza die Verhandlungsposition der griechischen Regierung mit dem Argument stärken, wenn die Troika die Sparziele nicht entschärft, kommt eine Euro-feindliche Regierung zustande. Umgekehrt kann Bundeskanzlerin Merkel mit Verweis auf die Abweichler in der eigenen Fraktion deutlich machen, dass die Zahlungsbereitschaft ihrer Wählerschaft nicht grenzenlos ist. Irgendwann könnte jedoch analog zur britischen Austrittsdrohung eine ungewollte Dynamik in Gang gesetzt werden, die zum Zerbrechen der Währungsunion führen könnte. Denn natürlich möchte auch Ministerpräsident Cameron im gemeinsamen Binnenmarkt bleiben; aber was zunächst als cleverer Schachzug zur innerparteilichen Befriedung schien - eine Volksabstimmung über Britanniens Verbleib in der EU zu versprechen - könnte durchaus zum ungewollten Ergebnis führen.

\section{Die offenen Flanken des europäischen Einigungswerks überwinden: Abkürzung durch Souveränitätsverzicht?}

In den Augen der Befürworter einer beschleunigten EU-Europäisierung als Krisenlösung gibt es nur die Alternative >mehr EU< oder das Scheitern Europas. Ein Kompetenztransfer hin zu den EU-Gremien zur Kontrolle der Haushalts- und zur Koordinierung nationaler Wirtschaftspolitiken könnte, so die Überlegung, die offenen Flanken des europäischen Einigungswerks überwinden. Während die Bundesregierung sauf Sicht< (Merkel) fährt und so eine immer kompliziertere Gemengelage aus multilateralen und supranationalen Institutionen mit teils eigenverantwortlichen Rechtspersönlichkeiten erzeugt, soll mittel eines sgroßen Integrationsschrittes die Weichen für eine Politische Union gestellt werden. ${ }^{15}$ Dass die Bürger diese Selbstermächtigung der Politik ${ }^{16}$ mehrheitlich ablehnen, sei hinzunehmen, da die Souveränitätsübertragung die zugegebenermaßen bislang noch schwachen demokratischen Grundlagen der Europäischen Union grundlegend stärke. ${ }^{17}$

Dieses Modell besitzt theoretischen Charme. Es vermindert die Durchsetzungsproblematik, da die Aufsicht über die nationale Haushaltspolitik nun von demokratisch le-

15 Peter Bofinger / Jürgen Habermas / Julian Nida-Rümelin, »Kurswechsel für Europa. Einspruch gegen die Fassadendemokratie in: Frankfurter Allgemeine Zeitung, 3.8.2012.

16 Bofinger / Habermas / Nida-Rümelin, Kurswechsel für Europa, aaO. (FN 15).

17 Ulrich Beck, Das deutsche Europa. Neue Machtlandschaften im Zeichen der Krise, Berlin 2012, Daniel Cohn-Bendit / Guy Verhofstadt, Für Europa! Ein Manifest, München 2012 und durchaus differenzierter - Martin Schulz, Der gefesselte Riese, Berlin 2013. 
gitimierten Institutionen erfolgen soll. Die institutionellen Schwächen der Währungsunion - die fehlende Steuer-, Haushalts- und Wirtschaftspolitik - würden korrigiert und durch einen umfangreichen Solidarmechanismus, sprich Transferunion, sozial abgesichert werden. Das angedachte föderale Europa soll demokratisch, handlungsfähig und dann auch wieder bürgernah sein.

Ein nüchterner Blick auf Europas politisches Einigungswerk im Zeitablauf lässt erhebliche Zweifel an diesem Szenario aufgekommen. Ähnlich wie in der kommunistischen Utopie wird ein Modell des von seinen Mängeln gereinigten rreal existierende Europas ${ }^{18}$ in die Zukunft projiziert. Gewinner bisheriger Europäisierungsprozesse waren die Eliten vor allem in der Wirtschaft, ${ }^{19}$ aber auch in Politik und Verwaltung ${ }^{20}$ und in der Medienöffentlichkeit. ${ }^{21}$ Damit sollen keineswegs die vielen positiven Begleiterscheinungen des Integrationsprozess gering geachtet, noch die Mängel der Nationalstaaten alter Prägung übersehen werden. Doch war die europäische Integration mit derart vielen ungedeckten Hoffnungen verbunden, dass vermutlich auch die Realität eines einheitlichen föderalen Europa ganz anders aussehen dürfte, als es sich die Mehrheit seiner Befürworter ausmalt.

Die ökonomische Liberalisierung durch Europäisierung lässt sich gerade als Mit-Auslöser der Finanzkrise zeigen. Es war entgegen mancher Darstellung keineswegs so, dass die Finanzmärkte nicht Gegenstand umfassender Regulierung durch die EU gewesen wäre. ${ }^{22}$ Allerdings war diese, entsprechend den Forderungen der großen internationalen Finanzinstitute, hauptsächlich darauf ausgerichtet, durch Rechtsangleichung nationale Besonderheiten zu beseitigen und einen gemeinsamen europäischen Finanzmarkt zu schaffen. Beispielsweise wurde die Sonderstellung der deutschen Landesbanken beseitigt, was mit dazu beitrug, dass diese Landesbanken glaubten, auf den internationalen Finanzmärkten das große Rad drehen zu müssen. ${ }^{23}$ Es sind weniger die eklatanten Fehleinschätzungen der EU-Kommission zu Beginn der Finanzkrise - mit denen sie ja nicht allein steht - sondern ihr unerschütterlicher Glaube an die anglo-amerikanische Archi-

18 Stefan Immerfall, Europa - politisches Einigungswerk und gesellschaftliche Entwicklung, Wiesbaden 2006, S. 25.

19 Streeck, Gekaufte Zeit, aaO. (FN 4).

20 Max Haller, Die Europäische Integration als Elitenprozess. Das Ende eines Traums?, Wiesbaden 2009.

21 Ruud Koopmans / Paul Statham, The Making of a European Public Sphere. Media Discourse and Political Contention, Cambridge 2010.

22 Christian Schröder, Europa in der Finanzfalle. Irrwege internationaler Rechtsangleichung, Berlin 2012, S. 42-73.

23 Von Wall Street-Insidern als „Stupid Germans“ bekannt: Michael Lewis: The Big Short. Inside the Doomsday Machine, London 2010, S. 93. 
tektur der Finanzmärkte ${ }^{24}$ und vor allem ihr außergewöhnlich hartleibiger Mangel an Selbstkritik, ${ }^{25}$ die zu denken geben.

Hinzu kommt, dass die Lehren aus bisherigen regionalen Wachstumsprogrammen ernüchternd sind. Das gilt selbst innerhalb Deutschlands, wo nach über zwei Jahrzehnten mit jährlichen Nettozahlungen von durchschnittlich $80 \mathrm{Mrd}$. Euro die Angleichung der Lebensverhältnisse zwischen West- und Ostdeutschlands ins Stocken geraten ist. In Italien ist die Bilanz der wirtschaftlichen und sozialen Konvergenzanstrengungen noch schlechter. Ein entschlossener transeuropäischer Solidarausgleich würde angesichts der innereuropäischen Entwicklungsunterschiede Summen erfordern, die völlig jenseits der Vorstellungskraft und wohl auch der Geduld des deutschen (oder österreichischen und finnischen) Steuerzahlers sind. ${ }^{26}$

Auch wenn wir theoretisch angeleitete Prognosen heranziehen und ihre Voraussagen mit den dann eingetretenen Entwicklungen vergleichen, ergibt sich ein klares Bild: Friedrich von Hayek (1958) hat über Karl Deutsch (1966) 'gesiegt<, die Freiheit der Märkte über kontinentale Gemeinschaftsbildung. ${ }^{27}$ Beide gingen von einer friedenssichernden Föderation aus, in der wirtschaftliche Freiheit herrsche. Während Deutsch aufgrund zunehmender Transaktionen die Entwicklung eines nationenübergreifenden Gemeinschaftssinns erwartete, bezweifelte Hayek, dass sich auf der größeren Ebene umfangreiche Solidaritätsgefühlt entwickeln könnten. Er begrüßte dies auch, da die Abwesenheit einer gemeinsamen Identität wirtschafspolitische und sozial motivierte Eingriffe in das Marktgeschehen erschweren würde. Auf internationaler Ebene würden kaum die notwendigen Mehrheiten zustande kommen um - von Hayek als Anathema stets bekämpft - >soziale Gerechtigkeit gegen die Freiheit des Individuums in Anschlag zu bringen. Diese, von Hayek klarsichtig beschriebene Entwicklungslogik begünstigte die >Transformation der Europäischen Union in ein Vehikel der Liberalisierung des Europäischen Kapitalismus ${ }^{28}$ Das Plädoyer für einen >großen Integrationsschritt< überschätzt also vermutlich die steuerungstheoretische Kapazität der Politischen Union, unterschätzt seine neoliberale Schlagseite und missachtet die Grenzen der pragmatischen Verbundenheit, die das Gemeinschaftsprojekt bislang getragen hat.

24 Dies überrascht vielleicht weniger, wenn man bedenkt, dass zentrale Kommissionsmitglieder wie Mario Monti oder Romano Prodi wie auch der Präsident der Europäischen Zentralbank, Mario Draghi, Mitarbeiter bei Goldmann Sachs waren.

25 Für anschauliches Beispiel siehe Christian Schröder, Europa in der Finanzfalle. Irrwege internationaler Rechtsangleichung, Berlin 2012, S. 43, 45-48, 69); allg. Ewald Engelen et al., » Misrule of experts? The financial crisis as elite debacle« in: Economy and Society 41, Nr.3 (2012), S. 360-382.

26 Für Größenordnungen siehe Streeck, Gekaufte Zeit, aaO. (FN 4), S. 187-203.

27 Friedrich von Hayek, »The Economic Conditions of Interstate Federalism« in: ders. Individualism and Economic Order, Chicago 1958 [orig. 1939], S. 255-272, Karl W. Deutsch et al., »Political Community and the North Atlantic Area, in: K. W. Deutsch et al. (Hg.), International Political Communities. An Anthology, Garden City 1966, S. 1-91.

28 Zu Hayek siehe Streeck, Gekaufte Zeit, aaO. (FN 4), S. 141-148 (Zitat S. 147), zu Deutsch siehe Stefan Immerfall/Eckhard Priller/Jan Delhey, »Associations and Community «, in: Immerfall, Stefan und Göran Therborn (Hg.), Handbook of European Societies. Social Transformations in the 21st Century. New York, S. 7-37, hier S. 25-33. 


\section{Die gesellschaftlichen Grenzen Europas und die Grenzen der Politischen Union}

Aus soziologischer Sicht ist darauf hinzuweisen, dass das Fehlen einer europäischen Gesellschaft eine entscheidende Rahmenbedingung darstellt, die zu übersehen das europäische Projekt - das man doch retten will - gefährden könnte. Wenn die Diagnose stimmt, dass das Fehlen einer europäischen Gesellschaft die Logik des Integrationsprozess mitbestimmt, liegt es nahe, den gut gemeinten Zentralisierungs- und Angleichungsbemühungen der Europäischen Sozialingenieure und den Schönmalern des postnationalen Europas skeptisch gegenüber zu stehen. Angesichts der widerstreitenden Vielfalt und der schwach ausgeprägten Identität Europas erscheint es fraglich, ob die Bürger in den Geberländern wie auch in den Nehmerländern zu dem mit einer europäischen Wirtschaftsregierung verbundenen Souveränitätsverzicht bereit wären, selbst wenn sie mehr demokratische Legitimität besäße, als das heute der Fall ist.

Das Fehlen einer europäischen Gesellschaft äußert sich in erster Linie in dünnen Vertrauensbeziehungen der Mitgliedsgesellschaften untereinander und in der (nur) bedingten Loyalität zur supranationalen Einheit. ${ }^{29}$ In guten Zeiten wurden die gesellschaftlichen Grundlagen der europäischen Integration lang übersehen oder als unwichtig erachtet. Nun wird eingewandt, dass die EU gar kein Gemeinsamkeitsgefühl nach Art des nationalen Gemeinschaftsgefühls brauche. Die EU sei ein postnationales oder gar kosmopolitisches Projekt. ${ }^{30}$ Dieser Einwand übersieht, dass die europäischen Identitäten national gespeiste Deutungen sind. Sie koexistieren scheinbar harmonisch mit nationalen (in einigen Ländern wie Spanien auch subnationalen) Zugehörigkeitsgefühlen - aber nur solange die endgültige Gestalt der Gemeinschaft ungeklärt bleibt und solange Gewinner und Verlierer in der europäischen Arena nicht feststehen.

Charakteristikum der Europäischen Union ist, dass sich ihre Mitgliedsstaaten freiwillig verpflichten, dem Recht der Europäischen Union auf ihren Territorien unter der Maßgabe Geltung zu verschaffen, dass alle anderen Mitgliedsländer dies auch tun. ${ }^{31}$ Wohlfahrtsgewinne durch Selbstbindung, nicht Unterwerfung unter eine noch so gut gemeinte zentrale Gestaltungsmacht, entspricht dem europäischen Gedanken. Mit den verschiedenen Rettungspaketen und der Ankündigung der Europäischen Zentralbank, in einer Zwangslage notleidende Staatsanleihen zu kaufen, haben die EU-Eliten Zeit gekauft. Aber sie können nicht auf Dauer Technokraten als Landverwalter in die Schuldenländer schicken. Wenn der Weg zu einer supranationalen Lösung versperrt - oder zumindest nicht empfehlenswert ist, sofern das Ganze nicht gefährdet werden soll - wäre es umso wichtiger, den Subsidiaritätsgedanken wieder stark zu machen.

29 Stefan Immerfall / Klaus Boehnke / Dirk Baier, »Identity « in: Stefan Immerfall / Göran Therborn (Hg.), Handbook of European Societies. Social Transformations in the 21st Century, New York 2010, S. 325-354.

30 Ulrich Beck / Edgar Grande, Gesellschaft und Politik in der Zweiten Moderne, Frankfurt/M. 2004.

31 Maurizio Bach »Jenseits der Souveränitätsfiktion. Der Nationalstaat in der Europäischen Union « in: Zeitschrift für Politik, Sonderband 5 (2013), S. 105-124, Stefan Immerfall, »Was hält die EU-Gesellschaften zusammen?« in: Stefan Köppl (Hg.), Was hält Gesellschaften zusammen? Ein internationaler Vergleich, Wiesbaden 2013, S. 109-121.

ZfP 60. Jg. 2/2013 
Angesichts des dünnen Reservoirs an gefühlten Gemeinsamkeiten in der und für die EU sollten Wege beschritten werden, den europäischen Integrationsprozess zu bewahren, ohne immer höhere Dosen an Vergemeinschaftung zu verabreichen. Es wäre sogar darüber nachzudenken, inwiefern die europäische Verflechtung nicht ein Stück weit zurückgenommen werden sollte. Namentlich die vergemeinschaftlichte Geldpolitik erschwert die wirtschaftliche Anpassung und Schuldentragfähigkeit der Mitglieder der Währungsunion in einem Maße, dass selbst eine möglichst geordnete Rückkehr zu einer verbesserten Version des früheren Europäischen Währungssystems ${ }^{32}$ nicht völlig ausgeschlossen werden sollte. Aber vorher sollte überlegt werden, wie die Instrumente der makroökonomischen Politik (insbesondere der Zinspolitik) auch im Rahmen der Währungsunion wieder stärker nationalen Besonderheiten Rechnung tragen könnten ${ }^{33}$.

EU-Kompetenzen sind auf Bereiche zu begrenzen, die den Bürgern einsichtig sind. Das ist in erster Line eine Gemeinschaftsrechtsordnung, die den Wirtschaftsegoismus der Mitgliedsstaaten bändigen und dadurch den Gesamtnutzen mehrt. Weitere Themen, in denen die Mitgliedsstaaten - oder besser: ihre Bevölkerungen - gemeinsame Interessen haben, sind die vielfältigen steuerlichen Gestaltungsspielräume im europäischen Binnenmarkt einerseits, die soziale fragwürdige Auslegung der Freizügigkeitsregeln unter anderem durch den Europäischen Gerichtshof ${ }^{34}$ andererseits. Die ersatzlose Aushöhlung der nationale Steuerhoheit im Prozess der Europäisierung begünstigt die Steuergestaltung für private Vermögen, exorbitante Einkommen und internationale Firmen ${ }^{35}$ in einem Maße, das angesichts der in allen Euro-Ländern stark gestiegenen Ungleichheit von Einkommen und Vermögen nicht mehr hingenommen werden sollte. Es gilt den Blick auf legale Steuerverkürzungspraktiken multinationaler Konzerne, ${ }^{36}$ die zahlreichen und

32 Fritz W. Scharpf, »Die Eurokrise: Ursachen und Folgerungen« in: Zeitschrift für Staats- und Europawissenschaften 9, Nr. 3 (2011) S. 324-337.

33 Vgl. z. B. Rainer Willi Maurer, »One Interest Rate for All - Has the European Central Bank Enough Instruments? in: SSRN, January 2011 (Available at http://papers.ssrn.com/sol3/papers.cfm?abstract_id=1797084).

34 Der Europäische Gerichtshof hat wiederholt nationale Schutzbestimmungen und Tarifvereinbarungen als > mit dem Binnenmarkt nicht vereinbar< verworfen (etwa im Falll "Rüffert" zur Europarechtswidrigkeit von Tariftreueklauseln; EuGH, 3.4.2008 - C-346/06). Mit dieser Rechtssprechung wurden Tür und Tor für ein Missbrauchs- und Ausbeutungssystem geschaffen, das insbesondere die Erweiterungsländer Bulgarien und Rumänien einbezieht, obgleich für diese Länder die volle Arbeitnehmerfreizügigkeit erst ab 2014 gilt. Vgl. Frankfurter Allgemeine Zeitung vom 15.4.2012, "Bulgaren in Deutschland. Alles was kommt." [http://www.fa z.net/aktuell/politik/inland/bulgaren-in-deutschland-alles-was-kommt-11716710.html; 7.4.2013] oder "Handwerker-Betrug? Geisterhaus: 60 Firmen gemeldet, niemand da", Express (Düsseldorf) vom 4.3.2013 [http://www.express.de/duesseldorf/handwerker-betrug--geisterh aus--60-firmen-gemeldet--niemand-da,2858,21994892.html; 7.4.2013].

35 Martin Höpner / Armin Schäfer (Hg.), Die politische Ökonomie der europäischen Integration, Frankfurt a.M. 2008.

36 Beispielsweise durch die Konstruktion eines "Double Irish With a Dutch Sandwich", s. New York Times, April 28, 2012 [http://www.nytimes.com/interactive/2012/04/28/business/Dou ble-Irish-With-A-Dutch-Sandwich.html; 7.4.2013]. 
ebenfalls legalen Begünstigungen von Großverdienern ${ }^{37}$ und die bislang völlig unglaubwürdige Kontrolle der Geldabflüsse in Steueroasen zu lenken, aber auch auf die im historischen Vergleich allgemein niedrigen Grenzsteuersätze und Vermögenssteuern.

Angesichts ihrer engen Verflechtung mit den Vermögenden und der Finanzindustrie tut sich die Politik mit entsprechenden Maßnahmen schwer. Etwas anderes erscheint derzeit realistischer, nämlich dass die Arbeitnehmer in den Exportländern der Eurozone nach Jahren der Stagnation wieder Reallohnsteigerungen einfordern. Damit würde sie ihren Anteil an den Produktivitätssteigerungen der letzen Jahrzehnte einklagen und zugleich die Wettbewerbsposition der Südländer verbessern helfen. Die Europäische Integration ist zu wichtig, um sie den Euro-Rettern zu überlassen.

\section{Zusammenfassung}

Die Euro-Krise ist Bestandteil der Krise des Finanzkapitalismus, aber sie hat auch spezifisch europäische Wurzeln. Dazu zählen nicht nur die großen Produktivitätsunterschiede zwischen den Euro-Ländern und die institutionelle Ausgestaltung der Währungsunion, sondern auch das Fehlen einer europäischen Gesellschaft. Im Europäischen Gemeinschaftssystem zwingt der eingeschlagene Konsolidierungskurs seine Mitgliedsländer in wechselseitige Konfliktsituationen, die die Währungsunion zu sprengen drohen. Aber ein >großer Integrationsschritt< würde die Grenzen der pragmatischen Verbundenheit, die das Gemeinschaftsprojekt bislang getragen hat, noch mehr missachten und überdies die steuerungstheoretische Kapazität der dann geschaffenen Politischen Union überschätzen. Angesichts des dünnen Reservoirs an gefühlten Gemeinsamkeiten in und für die EU sollten vielmehr Wege beschritten werden, den europäischen Integrationsprozess zu bewahren, ohne immer höhere Dosen an Vergemeinschaftung zu verabreichen. Es wäre sogar darüber nachzudenken, inwiefern die europäische Verflechtung nicht ein Stück weit zurückgenommen werden könnte.

\section{Summary}

The euro-crisis is part and parcel of the crisis of financial capitalism. Yet, it has its own European flavour. Differences in productivity and institutional deficits of the monetary union are important ingredients of the current predicament but even more fundamental is the peculiar absence of a European society. Current consolidation efforts within the European system set its members against each other thus threatening to break up the monetary union. The call for >more Europe is no way out of the conundrum either, because it misunderstands the pragmatic and instrumental nature of European integration and overestimates the steering capacity of the proposed Union. Given the weak ties

37 In Griechenland ist die Steuerbefreiungen für Reeder sogar in der Putschisten-Verfassung von 1967 festgeschrieben, vgl. Süddeutsche Zeitung vom 6. Dezember 2012 [http://www.sueddeu tsche.de/wirtschaft/griechische-reederfamilien-geraubte-schaetze-1.1543049; 7.4.2013].

ZfP 60. Jg. 2/2013 
within the European Union, the article argues for strengthening the principle of subsidiarity.

Stefan Immerfall, Via Euro-crisis to fiscal union? Potential lessons for the European integration

\section{Gemeinden als Fundament der EU?}

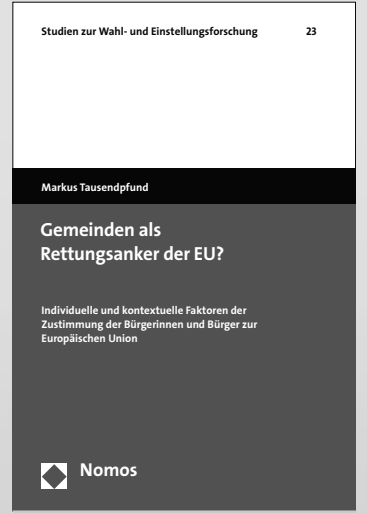

Ty nomoz
Gemeinden als Rettungsanker der EU?

Individuelle und kontextuelle Faktoren der Zustimmung der Bürgerinnen und Bürger zur Europäischen Union Von Markus Tausendpfund 2013, 410 S., brosch., 64,-€ ISBN 978-3-8487-0023-3

(Studien zur Wahl- und

Einstellungsforschung, Bd. 23)

Gemeinden gelten als "Keimzelle für das Zusammenleben in Europa“ und als „Fundament der Staatengemeinschaft". Für diese These findet sich aber keine empirische Überprüfung. Die Studie schließt diese Forschungslücke und untersucht erstmals den Einfluss individueller Merkmale und des lokalen Umfelds auf die Zustimmung der Bürger zur EU. 